\title{
The Role of Hepcidin in Iron Metabolism
}

\author{
Elizabeta Nemeth Tomas Ganz \\ David Geffen School of Medicine, University of California, Los Angeles, Calif., USA
}

\section{Key Words}

Anemia of inflammation - Bone morphogenetic protein • Hemochromatosis · Hepcidin • Iron-loading anemia

\begin{abstract}
Hepcidin is the central regulator of systemic iron homeostasis. Dysregulation of hepcidin production results in a variety of iron disorders. Hepcidin deficiency is the cause of iron overload in hereditary hemochromatosis, iron-loading anemias, and hepatitis C. Hepcidin excess is associated with anemia of inflammation, chronic kidney disease and iron-refractory iron deficiency anemia. Diagnostic and therapeutic applications of this new knowledge are beginning to emerge. Dr. Ernest Beutler played a significant role in advancing our understanding of the function of hepcidin. This review is dedicated to his memory.

Copyright $\odot 2009$ S. Karger AG, Basel
\end{abstract}

\section{Introduction}

This review is dedicated to the memory of Dr. Ernest Beutler who had a career-long interest in iron homeostasis and its relationship to erythropoiesis. Guided by his legendary knowledge of all aspects of hematology and careful analysis of experimental and clinical data, he contributed much to modern hematology and was not afraid to challenge established beliefs. Even after he fell termi- nally ill, he continued to make major contributions to the study of iron regulation. His ideas, attitudes, and achievements inspired us all.

\section{Hepcidin Synthesis and Catabolism}

The hormone hepcidin, a 25-amino-acid (aa) peptide, is the principal regulator of iron absorption and its distribution to tissues. Hepcidin is synthesized predominantly in hepatocytes, but its low levels of expression in other cells and tissues, including macrophages, adipocytes and brain, may also be important for the autocrine and paracrine control of iron fluxes at the local level.

Hepcidin is encoded as an 84-aa prepropeptide, containing an $\mathrm{N}$-terminal 24 -aa endoplasmic reticulum-targeting signal sequence. The 60 -aa prohormone contains a consensus furin cleavage motif, and several proprotein convertases were reported to process hepcidin in vitro including furin, PACE4, PC5/6 and PC7/LPC. The processing step occurs in the Golgi apparatus, does not appear to be regulated, and only the mature peptide, but not the prohepcidin, was shown to be secreted from cells [1].

The mature hormone circulates in plasma and its binding to $\alpha_{2}$-macroglobulin has been reported [2]. While this interaction was shown to promote hepcidin activity in vitro, the effect on hepcidin clearance is still unknown. A major route of hepcidin clearance is renal excretion. When kidney function is normal, urinary hepcidin con-

\section{KARGER}

๑ 2009 S. Karger AG, Basel

Fax +4161306 1234

E-Mail karger@karger.ch

www.karger.com
Accessible online at: www.karger.com/aha
Elizabeta Nemeth

Department of Medicine, UCLA

10833 LeConte Avenue, CHS 37-131

Los Angeles, CA 90095 (USA)

Tel. +1 310825 7499, Fax +1 310206 8766, E-Mail enemeth@ mednet.ucla.edu 


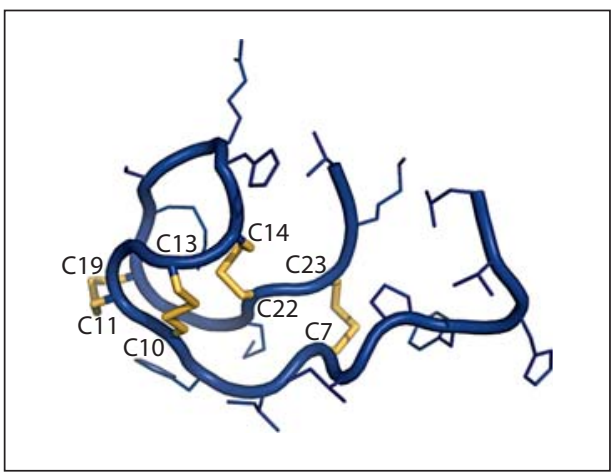

Fig. 1. Hepcidin structure with revised disulfide connectivities.

centrations correlate well with circulating hepcidin levels, with no apparent regulation of the excretion process. However, based on the comparison between serum and urinary concentrations, it appears that only $5 \%$ of hepcidin from plasma filtered in the kidneys ends up intact in the urine [3], suggesting that hepcidin may not be freely filtered in the glomerulus and/or that filtered hepcidin is reabsorbed and degraded in proximal tubules similarly to other small peptide hormones. Hepcidin may also be cleared by receptor-mediated endocytosis in tissues expressing its receptor ferroportin, as indicated by the accumulation of radiolabeled hepcidin in ferroportin-rich tissues [4] and the degradation of the endocytosed ferroportin-hepcidin complex in cultured cells. How much hepcidin catabolism occurs by renal clearance or by degradation in target tissues remains to be determined.

\section{Hepcidin Structure}

Structurally, the hepcidin peptide resembles a bent hairpin held together by four disulfide bonds. The disulfide connectivity was recently revised. NMR spectroscopy, partial reductive alkylation and Fourier transform mass spectroscopy were used to resolve ambiguities arising from the proximity of the four disulfides (Sasu et al., US patent application no. 2008/0213,277). The new model indicates that two bonds stabilize the antiparallel $\beta$-sheet, and two tether the bent conformation of the peptide (fig. 1). Our recent data indicate that hepcidin binding to its receptor requires the involvement of one of the disulfide bonds. However, considering that removal of individual bonds does not dramatically decrease hepcidin activity in vitro, multiple disulfide bonds must be capable of forming a contact with ferroportin.

Hepcidin
The disulfide bonding pattern is strictly conserved across species that produce hepcidin - fish, amphibians, reptiles and mammals. Moreover, hepcidin from one species can bind to the receptor from an evolutionarily distant species, e.g. human and zebrafish hepcidin were active against mouse ferroportin [5]. Apart from the disulfide bonds, structure-function studies also revealed that the N-terminus of hepcidin is important for its iron-regulatory activity. The N-terminally truncated human 20 aa peptide was inactive both in vitro and in vivo indicating that this region may also contain contact residues for hepcidin interaction with its receptor.

The amphipathic structure of hepcidin and its extensive disulfide bonding are common characteristics of antimicrobial and antifungal peptides. However, hepcidin has only displayed modest antimicrobial properties in vitro at very high concentrations $(10-30 \mu \mathrm{M})$, and the significance of its antimicrobial properties in vivo remains to be determined. Patients with hereditary hemochromatosis, a disease generally resulting from relative hepcidin deficiency, are reported to develop infections caused by unusual microorganisms (Vibrio, Yersinia and Listeria), but this susceptibility could be related to the bacteria benefitting from increased iron levels rather than from the loss of any direct antibacterial effect of hepcidin.

\section{Iron-Regulatory Activity of Hepcidin}

Hepcidin is the main regulator of plasma iron concentrations. Injection of hepcidin into mice resulted in a dramatic drop in serum iron within just $1 \mathrm{~h}$ [4]. Even though hepcidin is rapidly cleared from the plasma, the effect of a single dose was apparent for up to $72 \mathrm{~h}$, likely because of the time required to resynthesize sufficient amounts of the hepcidin receptor, ferroportin.

Chronic overexpression of hepcidin causes iron-restricted anemia in mice and humans, typically manifested as microcytic, hypochromic anemia. Conversely, hepcidin deficiency in mice and humans results in iron overload with iron deposition in the liver and other parenchyma, and sparing of the macrophage-rich spleen. Complete absence of hepcidin in humans causes juvenile hemochromatosis, the most severe form of hereditary hemochromatosis.

The phenotypes of hepcidin excess and deficiency indicate that hepcidin inhibits intestinal iron uptake and the release of iron from macrophages recycling old red blood cells (fig. 2). When hepcidin was overexpressed during embryonic development, fetuses developed severe

Acta Haematol 2009;122:78-86 


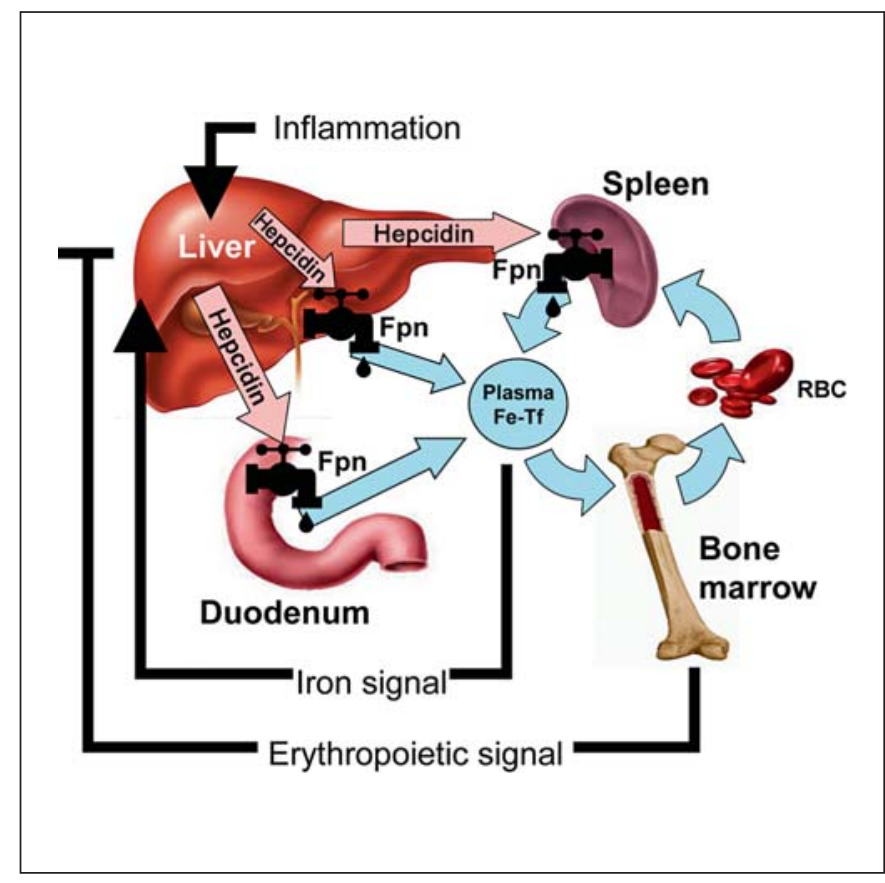

Fig. 2. Hepcidin-ferroportin (Fpn) interaction determines the flow of iron into plasma. Hepcidin concentration is in turn regulated by iron, erythropoietic activity and inflammation.

iron deficiency anemia and most died at birth indicating that hepcidin also inhibited the placental transport of iron [6]. Hepcidin also appears to block, at least partially, the export of stored iron from hepatocytes as indicated by hepatic iron accumulation in mice carrying hepcidinoverproducing tumors.

Hepcidin derived from extrahepatic sources may also exert control over local iron fluxes within tissues in which hepcidin is produced. For example, the central nervous system is separated from the plasma by the blood-brain barrier, and circulating hepcidin may not be transported across this barrier. However, brain tissue itself was reported to express hepcidin, allowing the possibility of iron regulation independent of the systemic control.

\section{Mechanism of Hepcidin Action}

Hepcidin acts by modulating cellular iron export through ferroportin to plasma and extracellular fluid (fig. 2). Ferroportin is both the hepcidin receptor and the only known cellular iron exporter in vertebrates. Ferroportin is expressed on cells that act as professional iron
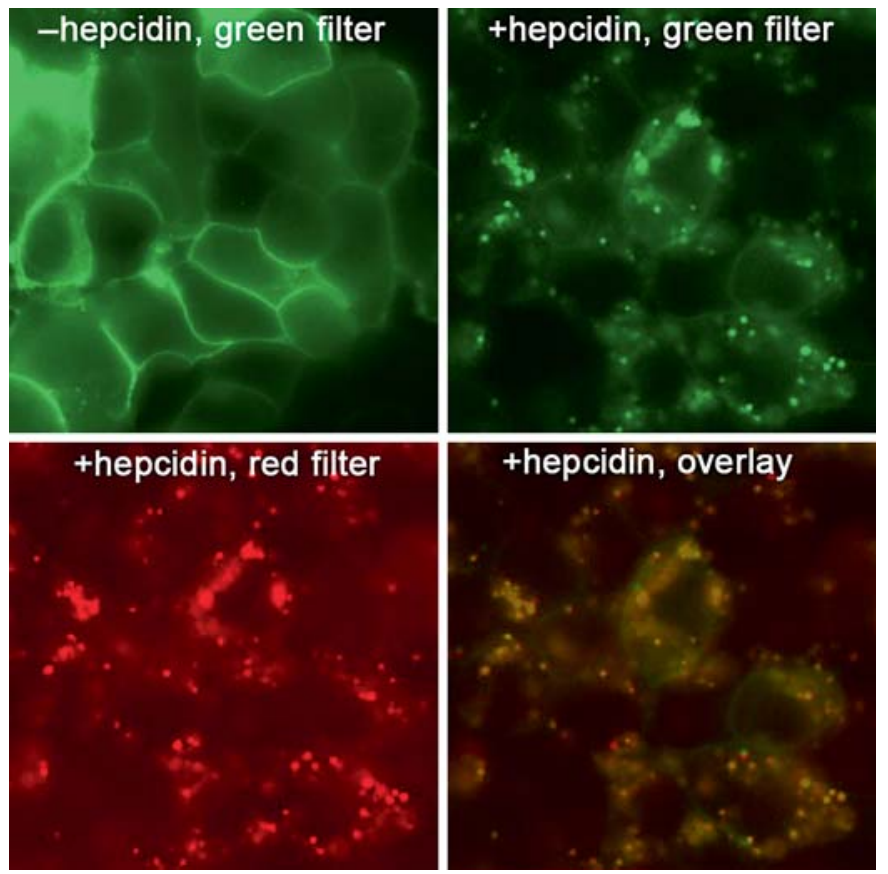

Fig. 3. Hepcidin binding to ferroportin causes internalization of the receptor-ligand complex, and their eventual degradation. HEK293 cells expressing ferroportin-GFP were treated with Texasred-labeled hepcidin and imaged using fluorescent microscopy.

handlers in the body: duodenal enterocytes absorbing dietary iron, macrophages in liver and spleen recycling old erythrocytes, hepatocytes storing iron and placental trophoblasts transferring iron to the fetus during pregnancy [7]. Ferroportin is also expressed in erythroid precursor cells, and it has been proposed that its presence enhances the sensitivity of precursors to systemic iron levels and helps determine their commitment to expansion and differentiation [8]. The complete loss of ferroportin expression in zebrafish and mouse models was shown to be embryonic lethal due to the inability of embryonic trophoblasts to transfer iron from the mother to the embryo. In the selective ferroportin knockout mice that preserved placental ferroportin, the newborn mice lacking ferroportin developed severe iron deficiency anemia due to low dietary iron absorption, and defective release of iron from hepatic storage and iron-recycling macrophages [7].

Posttranslational control of ferroportin levels by its ligand hepcidin is the major mode of ferroportin regulation. The binding of hepcidin to ferroportin triggers the internalization and degradation of the receptor-ligand complex [9] (fig. 3). The binding likely involves disulfide exchange between one of disulfide bonds of hepcidin and 
the exofacial ferroportin thiol residue Cys326. Patients with C326S mutations develop early-onset iron overload, and the mutant ferroportin lost its ability to bind hepcidin in vitro [10]. Once internalized, the hepcidin-ferroportin complex is degraded in lysosomes and cellular iron export ceases.

Ferroportin expression can also be regulated independently of hepcidin, by cellular iron content. Cellular iron has been shown to have an effect both at the transcriptional and translational level, the latter through a mechanism involving cytoplasmic iron-regulatory proteins and their corresponding binding sites in the $5^{\prime}$-region of one of the ferroportin mRNA isoforms [8].

Interestingly, the mechanism of iron transport by ferroportin has remained unexplored, and is one of the important challenges in advancing our understanding of cellular and systemic iron regulation.

\section{Regulation of Hepcidin}

Hepcidin is homeostatically regulated by iron and erythropoietic activity. Iron excess stimulates hepcidin production, and increased concentrations of the hormone in turn block dietary iron absorption thus preventing further iron loading (fig. 2). Conversely, hepcidin is suppressed in iron deficiency, allowing increased absorption of dietary iron and replenishment of iron stores. Increased erythropoietic activity also suppresses hepcidin production. Apart from enhancing iron absorption, this enables the rapid release of stored iron from macrophages and hepatocytes and augments the supply of iron for erythropoiesis. The molecular mechanisms underlying hepcidin regulation by iron and erythropoiesis are areas of intense investigation but are still incompletely understood. Hepcidin is also increased in inflammation and infection, and it is presumed that this regulation evolved as a host defense strategy to limit iron availability to microorganisms.

\section{Hepcidin Regulation by Iron}

Hepcidin is likely regulated by both circulating iron transferrin (Tf) and intracellular iron stores. Although the iron-sensing molecules for extracellular versus intracellular iron seem to be different, they both appear to utilize the bone morphogenetic protein (BMP) pathway to alter hepcidin expression. The BMP pathway has emerged as the critical regulator of hepcidin expression. BMPs control a variety of biological processes during embryonic and postnatal development, and signal by bind- ing to several types of BMP receptors. This results in phosphorylation of cytoplasmic Smad1/Smad5/Smad8 which associate with the common mediator Smad4 and translocate into the nucleus where they act as transcription factors. Liver-specific disruption of Smad4 in mice resulted in iron overload due to severely decreased hepcidin expression [11]. Typically, BMP signaling is modulated by coreceptors, among which hemojuvelin (HJV) is specific for iron regulation. Humans with disruptive HJV mutations or HJV knockout mice have iron overload as severe as that caused by ablation of hepcidin, with no other apparent problems [12].

In addition to the BMP/HJV pathway, other factors are necessary for hepcidin regulation by iron Tf. These include Tf receptor 2 (TfR2) and HFE, two molecules which are mutated in the adult forms of hereditary hemochromatosis. TfR2 is a homolog of TfR 1 , the receptor essential for the uptake of iron Tf by erythrocytes, and also expressed on most other cell types. TfR 2 is primarily expressed in the liver, and while its mRNA expression is not affected by iron levels, its iron-sensing function appears to involve the stabilization of TfR2 protein after binding of iron Tf [13]. HFE, a protein similar to MHC class I molecules, does not bind iron directly but instead interacts with iron-binding proteins, TfR1 and TfR2. It has been proposed that HFE shuttles between TfR 1 and TfR 2 depending on iron $\mathrm{Tf}$ concentrations [14]. In the absence of iron Tf, HFE associates with TfR1, but when iron Tf binds to TfR1, HFE is displaced and associates with TfR2 instead. Gao et al. [15] recently demonstrated that interaction of HFE and TfR2 is required for hepcidin induction in response to iron Tf. How the HFE/TfR2 complex signals to the hepcidin promoter is still unclear, but it appears to involve the BMP pathway. Inhibitors of BMP signaling (noggin and sHJV) prevented hepcidin mRNA increase after holo-Tf treatment in primary hepatocytes in vitro [16]. Because HFE and TfR2 were reported to interact with HJV, it is possible that binding of iron Tf to TfR1 and TfR2 initiates the formation of a supercomplex composed of HFE, TfR2, HJV and BMP receptors (fig. 4).

Apart from iron Tf, hepcidin may also be regulated by iron stores, presumably by some form of intracellular iron. HFE and TfR2 are not required for this regulation as mice and humans with HFE and TfR2 mutations are still capable of decreasing hepcidin levels after iron depletion. Although the mechanism of intracellular iron sensing is unclear, new evidence suggests that it may center on BMP6 [17]. First, BMP6 mRNA expression was shown to be regulated by iron loading: mice fed for 3 weeks with low-iron $(<3 \mathrm{ppm})$ or high-iron $(8,300 \mathrm{ppm})$ 


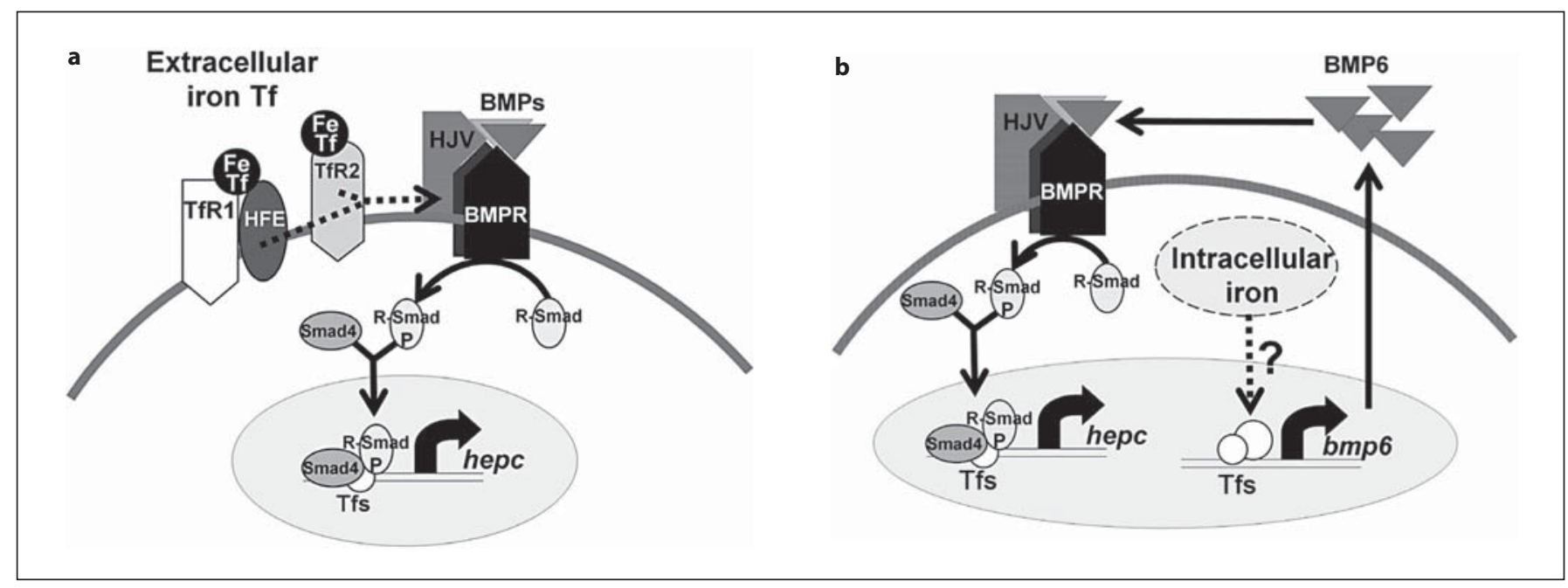

Fig. 4. A model of hepcidin regulation by iron Tf (a) and intracellular iron (b). a Increased iron Tf concentrations stabilize TfR2 protein, HFE is displaced from TfR1 and associates with TfR2. The HFE-TfR2 complex likely interacts with the HJV-BMP recep-

diet had lower and higher BMP6 mRNA, respectively, than mice fed an iron-balanced diet (200 ppm). Moreover, BMP6 protein was shown to interact directly and specifically with the coreceptor HJV. Finally, the disruption of BMP6 in mice resulted in very low expression of hepcidin and severe iron overload, but no other significant abnormalities. While these studies highlight the specific role of BMP6 in hepcidin regulation, it remains to be determined how the production of BMP6 itself is regulated by iron.

Additional molecules were shown to interact with HJV and modify its cell surface expression, including a protease (TMPRSS6) and a large multifunctional transmembrane protein (neogenin), but whether these processes are regulated by iron or other signals remains to be determined.

\section{Hepcidin Regulation by Erythropoietic Activity}

Hepcidin is decreased in iron deficiency anemia, hemolytic anemia, and anemias with ineffective erythropoiesis. The mechanisms mediating hepcidin suppression in these conditions, however, may not be the same. While the molecular details of the pathways involved are unclear, they may include soluble protein(s) produced by the developing erythroblasts in the bone marrow, decreased circulating or stored iron, and hypoxia.

In mouse models of anemias caused by bleeding or hemolysis, two studies showed that hepcidin suppression tor (BMPR), BMP signaling is potentiated and hepcidin transcription increases. b Elevated intracellular iron increases BMP6 production. Secreted BMP6 binds to the HJV-BMPR complex, stimulates Smad signaling, and increases hepcidin transcription. was dependent on intact erythropoietic activity. When erythropoiesis was inhibited using cytotoxic agents or irradiation, hepcidin mRNA did not decrease despite severe anemia [18].

Two proteins produced by erythroid precursors, growth differentiation factor 15 (GDF15) and twisted gastrulation protein (TWSG1), have been proposed to mediate hepcidin suppression in anemias with ineffective erythropoiesis [19]. GDF15 is a member of the TGF- $\beta$ superfamily, and although GDF15 knockout or transgenic mice do not have obvious iron abnormalities, larger doses of GDF15 do suppress hepcidin mRNA in vitro. Such doses are comparable to the levels of GDF15 observed in $\beta$-thalassemia and congenital dyserythropoietic anemia type I, diseases with massive overproduction of GDF15.

TWSG1 is a BMP-binding protein produced early during erythroblast maturation. TWSG1 also suppresses hepcidin in vitro, and it acts by interfering with BMP-dependent hepcidin regulation. Not much is known about TWSG1 expression in humans, but TWSG1 expression was increased in a mouse model of thalassemia.

Hepcidin is decreased by hypoxia, but the physiological relevance of this regulation is still uncertain. Alterations in the hypoxia-inducible factor (HIF) pathway in vivo can affect hepcidin expression. Liver-specific stabilization of HIF in mice, achieved by disruption of von Hippel-Lindau (VHL), resulted in markedly decreased hepcidin mRNA levels [20]. When these mice had an ad- 
ditional gene knocked out encoding a component of the HIF transcriptional complex, hepcidin levels were not suppressed any more. Whether HIF directly regulates hepcidin transcription is still unresolved. One study showed that HIF-1 bound to the mouse and human hepcidin promoters, but another study found that overexpression or knockdown of HIF-1 did not alter hepcidin expression in HepG2 cells. The indirect effects of hypoxia may be more important. Hypoxia-induced erythropoietin (EPO) could act as an intermediate and suppress hepcidin production through EPO-stimulated proliferation of erythroblasts, secreted products of erythrocyte precursors, and eventually increased iron utilization for hemoglobin synthesis.

\section{Hepcidin Regulation by Inflammation}

Hepcidin synthesis is rapidly increased by infection and inflammation. Of the specific pathways, IL- 6 was shown to be a prominent inducer of hepcidin, through a STAT3-dependent transcriptional mechanism. In human volunteers infused with IL-6, urinary hepcidin excretion was increased several fold within $2 \mathrm{~h}$ after infusion [21]. In mice, other cytokines can also directly regulate hepcidin production, since IL-6 knockout mice with chronic inflammation had elevated hepcidin mRNA similarly to wild-type mice. IL-1 was shown to increase hepcidin mRNA in mouse hepatocytes independently of IL-6, but it remains to be determined whether there are any species-specific differences in hepcidin regulation by inflammation.

\section{Hepcidin and Diseases}

Inappropriate production of hepcidin contributes to the pathogenesis of various iron disorders (table 1). Relative deficiency in hepcidin is associated with development of iron overload, whereas relative excess of hepcidin causes iron restriction and anemia.

\section{Diseases of Hepcidin Deficiency}

Hepcidin deficiency results in the development of systemic iron overload because of excessive iron absorption. In the absence of hepcidin, ferroportin concentrations on the basolateral surface of enterocytes are increased, leading to enhanced transport of dietary iron into plasma. In hepcidin deficiency, macrophages also display increased ferroportin on their cell membranes and thus export more iron. Excess plasma iron accumulates in organs in which iron uptake exceeds the rate of iron export. The
Table 1. Iron disorders associated with inappropriate hepcidin production

\begin{tabular}{ll}
\hline Defect in hepcidin production & Disease \\
\hline Hepcidin deficiency & $\begin{array}{l}\text { hereditary hemochromatosis } \\
\text { iron-loading anemias } \\
\text { hepatitis C }\end{array}$ \\
\hline Hepcidin excess & $\begin{array}{l}\text { anemia of inflammation } \\
\text { chronic kidney diseases } \\
\text { iron-refractory iron deficiency } \\
\text { anemia }\end{array}$ \\
\end{tabular}

liver is most commonly affected by iron overload due to the avid uptake of non-Tf-bound iron by hepatocytes. Iron overload of other organs appears to correlate broadly with the rate of iron absorption. Rapid accumulation of iron, such as seen with severe hepcidin deficiency (juvenile hemochromatosis or thalassemia intermedia), is associated with prominent deposition of iron in the heart and some endocrine organs. Although hepcidin deficiency is the common denominator of several different iron overload disorders, the molecular mechanisms that cause hepcidin deficiency are diverse.

In hereditary hemochromatosis [reviewed by Camaschella and Poggiali in this issue; pp 140-145], hepcidin deficiency results either from mutations in the hepcidin gene itself, or in the genes encoding hepcidin regulators. A very rare form of hemochromatosis is not associated with hepcidin deficiency, but with ferroportin resistance to hepcidin. Hepcidin regulators which are mutated in hereditary hemochromatosis include HFE, TfR2 and $\mathrm{HJV}$, the molecules involved in sensing of iron and subsequent signal transduction. Importantly, the degree of hepcidin deficiency correlates with the severity of iron overload, so that the most severe form of hemochromatosis, juvenile hemochromatosis, develops with mutations in the hepcidin or HJV genes, where hepcidin levels are completely or nearly absent. TfR 2 and HFE mutations result in a milder phenotype, particularly HFE mutations, which have a lower clinical penetrance.

Phlebotomy is currently the main treatment for patients with hereditary hemochromatosis. Although this is effective at removing excess iron, complete iron depletion appears to result in a further decrease in hepcidin levels which would be expected to enhance dietary iron absorption and increase the need for phlebotomy. Further studies are needed to establish whether less iron depletion, which would not lower hepcidin levels as much, 
would still be safe for patients and allow less frequent phlebotomy.

In iron-loading anemias, such as $\beta$-thalassemia and congenital dyserythropoietic anemias, urinary and serum hepcidin are severely decreased despite systemic iron overload [22]. The signal causing hepcidin suppression appears to be generated by high erythropoietic activity and outweighs the effects of iron overload on hepcidin regulation. As mentioned earlier, GDF15 and TWSG1 are two erythroid-produced factors that may contribute to hepcidin suppression in these syndromes. Transfusions increase hepcidin levels, presumably due to both the alleviation of ineffective erythropoiesis and increased iron load. Interestingly, patients with thalassemia intermedia were shown to have liver iron concentrations similar to those of regularly transfused thalassemia major patients, but because of the different hepcidin levels, the cellular distribution of iron in the liver was different. In thalassemia intermedia, similar to hereditary hemochromatosis, iron was heavily deposited in hepatocytes, whereas higher hepcidin levels in thalassemia major caused a shift of iron into macrophages. It is therefore possible that therapeutic hepcidin could be useful in thalassemia to shift the iron from the parenchyma to macrophages, where it is less toxic.

Hepcidin levels were also reported to be low in patients with chronic hepatitis $\mathrm{C}$, a disease frequently accompanied by hepatic iron overload, which worsens liver damage. The mechanism by which hepatitis $C$ virus suppresses hepcidin synthesis is not well understood, but was reported to include the virus-induced oxidative stress.

\section{Diseases of Hepcidin Excess}

Human syndromes of hepcidin overproduction suggest, and mouse models demonstrate, that elevated hepcidin is sufficient to cause hypoferremia and anemia [23]. Mice administered a single intraperitoneal injection of synthetic hepcidin developed hypoferremia within $1 \mathrm{~h}$ which lasted for almost 3 days [4]. Transgenic mice strongly overexpressing hepcidin during embryonic development developed severe microcytic, hypochromic anemia in utero [6], and weaker hepcidin overexpression caused mild-to-moderate anemia which was associated with iron-restricted erythropoiesis [23]. The phenotype develops from hepcidin-mediated inhibition of iron recycling and absorption. Decreased flow of iron into plasma results in hypoferremia, and because most of the iron in plasma is destined for the bone marrow, lower iron availability affects hemoglobin synthesis and erythrocyte production. In human disease, elevated hepcidin may contribute to anemia observed in inflammatory disorders, chronic kidney disease (CKD), hepcidin-producing hepatic adenomas and hereditary iron-refractory iron deficiency anemia (IRIDA).

In inflammatory disorders, hepcidin production is stimulated by increased cytokines, prominently including IL-6. Chronic hepcidin-mediated iron restriction would be expected to eventually lead to anemia of inflammation (AI) [for further information, see the review by Agarwal and Prchal in this issue; pp 103-108]. Elevated hepcidin was observed in rheumatologic diseases, inflammatory bowel disease, multiple myeloma, and critical illness, but whether hepcidin is a necessary factor in the pathogenesis of anemia in each of these disorders has not yet been established with certainty. Studies in mice moderately overexpressing hepcidin indicate that increased hepcidin not only causes iron restriction but also blunted erythropoietic response to EPO, characteristic of AI. Hepcidin does not appear to decrease red blood cell survival, another feature associated with AI. The role of hepcidin in the suppression of EPO, sometimes seen in $\mathrm{AI}$, is unclear. Studies of interventions that selectively reduce hepcidin will be necessary to determine how essential the role of hepcidin is in inflammation-induced anemia.

Hepcidin concentrations were reported to be increased in CKD patients [for further information, see the review by Silverberg et al. in this issue; pp 109-119]. This could be caused by inflammation which frequently accompanies CKD, but even patients without significant inflammation had elevated hepcidin levels which progressively increased with the severity of CKD. Because hepcidin is cleared, at least in part, by filtration in the kidney, decreased kidney function is the likely factor contributing to this phenomenon. Indeed, some studies reported an inverse correlation between glomerular filtration rate and serum hepcidin. It has been postulated that high hepcidin may be the reason for EPO resistance commonly observed in CKD patients. Two initial studies, however, reported no correlation between hepcidin-25 levels and EPO dose, raising questions about the usefulness of hepcidin as a predictor of patients' response to EPO. However, the ability of high EPO doses to suppress hepcidin synthesis may confound these studies.

IRIDA is a disease characterized by congenital hypochromic, microcytic anemia, refractory to treatment with oral iron, and only partially responsive to parenteral iron. The phenotype has been described almost 30 years ago, but its molecular basis has only recently been unraveled. IRIDA is caused by increased hepcidin production due to 
mutations in the hepcidin suppressor, TMPRSS6. The mechanisms are covered in more detail in the review by Lee [this issue; pp 87-96].

\section{Clinical Applications of Hepcidin}

\section{Diagnostics}

Considering that hepcidin is fundamentally involved in the pathogenesis of many iron disorders, measurements of hepcidin concentrations in biological fluids would be expected to facilitate the diagnosis of those diseases (table 1). The evaluation of the diagnostic potential of hepcidin has only recently become possible with the development of assays for bioactive mature hepcidin in serum and urine. The methodologies include competitive ELISAs using biotinylated or radioiodinated hepcidin as tracers, and several mass-spectrometry-based assays (matrix-assisted laser desorption ionization/time of flight/mass spectrometry, surface-enhanced laser desorption/ionization, and liquid chromatography coupled with tandem mass spectrometric) using as internal standards isotopically labeled hepcidin or shorter hepcidin mutants. Measurements of prohepcidin in serum have also been reported, but these did not correlate with mature hepcidin concentrations indicating that prohepcidin is inadequate as a substitute for measuring biologically relevant hepcidin.

Although promising, the utility of hepcidin for the diagnosis and prognosis of iron disorders needs to be evaluated in larger clinical studies.

\section{Therapeutics}

As most types of hereditary hemochromatosis are caused by hepcidin deficiency, hepcidin agonists should be useful for the prevention or treatment of these diseases. The proof of principle was demonstrated when iron overload was successfully prevented in HFE knockout mice made to transgenically express hepcidin [24]. In practice, however, many patients are first diagnosed with advanced iron overload. In that scenario, hepcidin agonists may be useful as adjuncts to therapeutic phlebotomy, where they would act to redistribute iron to macrophages and away from parenchymal cells, block further iron absorption and eventually eliminate the need for maintenance phlebotomy. A similar approach may be useful in hepatitis $C$ patients with hepatic iron overload.

In $\beta$-thalassemias and other iron-loading anemias, hepcidin agonists may help control intestinal iron absorption, which is the sole cause of iron overload in pa- tients not receiving transfusions. Unlike in hereditary hemochromatosis, an important consideration in this disease is whether hepcidin administration would negatively affect erythropoiesis. For the patients with transfusion-related iron overload, the utility of hepcidin agonists is not clear. It is possible that hepcidin could alter the distribution of iron from parenchyma to macrophages where it is less harmful.

Hepcidin antagonists would be expected to benefit patients with diseases of hepcidin excess, manifested as iron-restricted anemia and, eventually, as systemic iron deficiency. This use of hepcidin antagonists was conceptually validated in a mouse model of AI induced by injections of Brucella abortus. While mice injected with EPO alone showed little response, combination therapy using EPO and hepcidin-neutralizing antibody or hepcidin siRNA restored normal hemoglobin levels (Sasu et al., US patent application no. 2008/0213,277). Apart from those directly interfering with hepcidin activity, other agents which target pathways regulating hepcidin production have also been described. Dorsomorphin, a small-molecule inhibitor of BMP signaling, prevented hepcidin induction by iron in vivo [25]. Soluble HJV, also acting as an antagonist of BMP signaling, decreased hepcidin baseline expression in mice and concurrently increased liver iron content. Finally, in patients with inflammatory diseases, anemia may be responsive to anti-cytokine therapies such as anti-IL-6 antibody. Undoubtedly, future studies will assess the risks and relative benefits of these treatment approaches.

\section{Conclusions}

Since the first reports of hepcidin almost a decade ago, our understanding of the function of hepcidin in iron homeostasis has expanded tremendously, a development that Dr. Ernest Beutler contributed to and greatly enjoyed. Diagnostic and therapeutic applications of the new knowledge are beginning to emerge. These advances will improve the health and well-being of the many millions of patients with iron disorders.

\section{Acknowledgment}

This study was supported by a grant from the National Institutes of Health (R01 DK082717). 


\section{References}

$>1$ Valore EV, Ganz T: Posttranslational processing of hepcidin in human hepatocytes is mediated by the prohormone convertase furin. Blood Cells Mol Dis 2008;40:132138.

-2 Peslova G, Petrak J, Kuzelova K, Hrdy I, Halada P, Kuchel PW, Soe-Lin S, Ponka P, Sutak R, Becker E, Huang ML, Suryo RY, Richardson DR, Vyoral D: Hepcidin, the hormone of iron metabolism, is bound specifically to alpha-2-macroglobulin in blood. Blood 2009; 113:6225-6236.

>3 Ganz T, Olbina G, Girelli D, Nemeth E, Westerman M: Immunoassay for human serum hepcidin. Blood 2008;112:4292-4297.

$\checkmark 4$ Rivera S, Nemeth E, Gabayan V, Lopez MA, Farshidi D, Ganz T: Synthetic hepcidin causes rapid dose-dependent hypoferremia and is concentrated in ferroportin-containing organs. Blood 2005; 106:2196-2199.

$>5$ Nemeth E, Preza GC, Jung CL, Kaplan J, Waring AJ, Ganz T: The N-terminus of hepcidin is essential for its interaction with ferroportin: structure-function study. Blood 2006;107:328-333.

6 Nicolas G, Bennoun M, Porteu A, Mativet S, Beaumont C, Grandchamp B, Sirito M, Sawadogo M, Kahn A, Vaulont S: Severe iron deficiency anemia in transgenic mice expressing liver hepcidin. Proc Natl Acad Sci USA 2002;99:4596-4601.

$>7$ Donovan A, Lima CA, Pinkus JL, Pinkus GS, Zon LI, Robine S, Andrews NC: The iron exporter ferroportin/Slc40a1 is essential for iron homeostasis. Cell Metab 2005;1:191200.

$>8$ Zhang DL, Hughes RM, Ollivierre-Wilson H, Ghosh MC, Rouault TA: A ferroportin transcript that lacks an iron-responsive element enables duodenal and erythroid precursor cells to evade translational repression. Cell Metab 2009;9:461-473.
$>9$ Nemeth E, Tuttle MS, Powelson J, Vaughn MB, Donovan A, Ward DM, Ganz T, Kaplan $\mathrm{J}$ : Hepcidin regulates cellular iron efflux by binding to ferroportin and inducing its internalization. Science 2004;306:2090-2093.

10 Fernandes A, Preza GC, Phung Y, De Domenico I, Kaplan J, Ganz T, Nemeth E: The molecular basis of hepcidin-resistant hereditary hemochromatosis. Blood 2009;114 437-443.

11 Wang RH, Li C, Xu X, Zheng Y, Xiao C, Zerfas P, Cooperman S, Eckhaus M, Rouault T, Mishra L, Deng CX: A role of SMAD4 in iron metabolism through the positive regulation of hepcidin expression. Cell Metab 2005;2: 399-409.

12 Niederkofler V, Salie R, Arber S: Hemojuvelin is essential for dietary iron sensing, and its mutation leads to severe iron overload. J Clin Invest 2005;115:2180-2186.

13 Johnson MB, Chen J, Murchison N, Green FA, Enns CA: Transferrin receptor 2: evidence for ligand-induced stabilization and redirection to a recycling pathway. Mol Biol Cell 2007; 18:743-754.

14 Goswami T, Andrews NC: Hereditary hemochromatosis protein, HFE, interaction with transferrin receptor 2 suggests a molecular mechanism for mammalian iron sensing. J Biol Chem 2006;281:28494-28498.

$>15$ Gao J, Chen J, Kramer M, Tsukamoto H, Zhang AS, Enns CA: Interaction of the hereditary hemochromatosis protein $\mathrm{HFE}$ with transferrin receptor 2 is required for transferrin-induced hepcidin expression. Cell Metab 2009;9:217-227.

16 Lin L, Valore EV, Nemeth E, Goodnough JB, Gabayan V, Ganz T: Iron transferrin regulates hepcidin synthesis in primary hepatocyte culture through hemojuvelin and BMP2/4. Blood 2007;110:2182-2189.

17 Camaschella C: BMP6 orchestrates iron metabolism. Nat Genet 2009;41:386-388.
18 Pak M, Lopez MA, Gabayan V, Ganz T, Rivera S: Suppression of hepcidin during anemia requires erythropoietic activity. Blood 2006;108:3730-3735.

19 Tanno T, Bhanu NV, Oneal PA, Goh SH, Staker P, Lee YT, Moroney JW, Reed CH, Luban NL, Wang RH, Eling TE, Childs R, Ganz T, Leitman SF, Fucharoen S, Miller JL: High levels of GDF15 in thalassemia suppress expression of the iron regulatory protein hepcidin. Nat Med 2007;13:1096-1101.

20 Peyssonnaux C, Zinkernagel AS, Schuepbach RA, Rankin E, Vaulont S, Haase VH, Nizet V, Johnson RS: Regulation of iron homeostasis by the hypoxia-inducible transcription factors (HIFs). J Clin Invest 2007; 117:1926-1932.

21 Nemeth E, Rivera S, Gabayan V, Keller C, Taudorf S, Pedersen BK, Ganz T: IL-6 mediates hypoferremia of inflammation by inducing the synthesis of the iron regulatory hormone hepcidin. J Clin Invest 2004;113: 1271-1276

22 Nemeth E, Ganz T: Hepcidin and iron-loading anemias. Haematologica 2006;91:727732.

23 Roy CN, Mak HH, Akpan I, Losyev G, Zurakowski D, Andrews NC: Hepcidin antimicrobial peptide transgenic mice exhibit features of the anemia of inflammation. Blood 2007;109:4038-4044.

24 Nicolas G, Viatte L, Lou DQ, Bennoun M, Beaumont C, Kahn A, Andrews NC, Vaulont $\mathrm{S}$ : Constitutive hepcidin expression prevents iron overload in a mouse model of hemochromatosis. Nat Genet 2003;34:97-101.

$25 \mathrm{Yu}$ PB, Hong CC, Sachidanandan C, Babitt JL, Deng DY, Hoyng SA, Lin HY, Bloch KD, Peterson RT: Dorsomorphin inhibits BMP signals required for embryogenesis and iron metabolism. Nat Chem Biol 2008;4:33-41. 